\title{
Plurilinguisme à l'école et formation des enseignants : enjeux didactiques et pédagogiques
}

\section{Patricia Monjo et Rita Peix}

\section{(2) OpenEdition}

\section{Journals}

Édition électronique

URL : http://journals.openedition.org/trema/3167

DOI : $10.4000 /$ trema.3167

ISSN : 2107-0997

Éditeur

Faculté d'Éducation de l'université de Montpellier

Édition imprimée

Date de publication : 1 novembre 2014

Pagination : $1-5$

ISSN : 1167-315X

\section{Référence électronique}

Patricia Monjo et Rita Peix, « Plurilinguisme à l'école et formation des enseignants : enjeux didactiques et pédagogiques », Tréma [En ligne], 42 | 2014, mis en ligne le 01 novembre 2014, consulté le 23 septembre 2020. URL : http://journals.openedition.org/trema/3167 ; DOI : https://doi.org/10.4000/ trema.3167

Ce document a été généré automatiquement le 23 septembre 2020.

Trema 


\title{
Plurilinguisme à l'école et formation des enseignants : enjeux didactiques et pédagogiques
}

\author{
Patricia Monjo et Rita Peix
}

1 Le présent numéro s'inscrit dans le prolongement d'un ancien numéro de Tréma $\left(\mathrm{n}^{\circ} 28\right.$, 2007) intitulé Plurilinguisme et enseignement, centré alors sur les réponses institutionnelles et pédagogiques à la question du plurilinguisme et de son enseignement. Nous avons souhaité, quelques années plus tard, reprendre cette même problématique en accueillant dans notre revue un certain nombre de réflexions et travaux de recherche susceptibles de mettre en lumière une diversité d'approches ayant pour projet commun de comprendre la nature des enjeux liés à la notion de plurilinguisme et des mises en œuvre possibles à la fois à l'école primaire et sur le terrain de la formation des enseignants.

2 Sept années se sont écoulées entre les deux numéros, au cours desquelles la question de la mise en forme scolaire du plurilinguisme n'a cessé de prendre de l'ampleur et de susciter des propositions dont la diversité témoigne de la richesse d'une notion ouverte aux approches plurielles. L'école primaire constitue un lieu important pour le développement des compétences et des valeurs liées au plurilinguisme. Certaines questions se posent d'emblée : quelles nouvelles articulations envisager entre la langue socialement dominante et les langues étrangères et régionales présentes au sein des classes? Quelle proportion du temps scolaire consacrer respectivement à l'étude des langues et à l'utilisation de chaque langue comme médium, à l'étude des langues pour elles-mêmes et à l'utilisation de la diversité linguistique et culturelle comme support de développement de compétences civiques et sociales, interculturelles, etc. Comment mettre en pratique une didactique intégrée efficace ? Le rôle de la formation initiale et continue des enseignants apparaît dès lors déterminant pour faire évoluer à la fois les pratiques et les attitudes. Quelle place accorder dans cette formation à la perspective qui se dégage alors d'avoir à prendre en charge l'apprentissage d'élèves appelés de plus en plus à devenir des acteurs sociaux plurilingues? 
3 Les articles de ce numéro se répartissent selon 2 axes principaux. Le premier axe correspond aux articles traitant plus particulièrement du plurilinguisme à l'école primaire ; le deuxième aborde la question de la formation des enseignants.

\section{Partie 1 - Quelle place pour le plurilinguisme à l'école?}

En ouverture de ce numéro, l'article de Gilles Forlot nous invite à mettre le spectre de l'anglais à distance pour montrer dans quelle mesure cette langue - perçue communément comme LA langue à combattre - peut être au contraire envisagée comme une langue-pivot mise au service d'appropriations plurilingues et contribuer ainsi à renforcer le plurilinguisme. L'auteur aborde la question de l'anglais dans sa dimension de "langue passerelle » susceptible d'être un tremplin pour l'apprentissage des langues et d'assumer une fonction de soutien au développement du plurilinguisme.

5 C'est plus précisément l'éducation plurilingue et interculturelle que Marisa Cavalli met au cœur de sa réflexion et présente comme l'une des réponses possibles aux besoins de l'école et des élèves qui en sont les acteurs principaux. Face à la diversité des apprenants et de leurs répertoires et à la multiplicité des approches disponibles pour des enseignements plurilingues, l'école doit opérer des choix. C'est, selon l'auteure, en adoptant une double perspective, celle d'une politique linguistique d'établissement et celle de la construction curriculaire, que l'école pourra garantir à tous les élèves une éducation de qualité.

6 Michel Freiss, quant à lui, s'intéresse aux conditions de la mise en pratique d'une didactique plurilingue, soumise à la compréhension préalable du terme compétence. En effet, dans le cadre d'une politique européenne plurilingue qui intègre le CECRL et l'approche par compétences, il s'agit d'interroger la nature linguistique même que le terme de compétence recouvre. L'auteur s'emploie à explorer les thèses piagétiennes et chomskyennes sur la question pour mieux dégager l'interaction existante entre les compétences linguistiques et les compétences cognitives, soulignant ainsi l'intérêt d'une approche holistique de l'enseignement des langues.

$7 \quad$ Pierre Escudé nous présente une approche relevant de l'intercompréhension entre les langues parentes et les disciplines (dites) non linguistiques. Un certain nombre d'expérimentations en classes lui permet de tirer des conclusions sur l'utilisation de l'intercompréhension intégrée auprès du public de cycle 3 : quelles compétences sont mises en jeu, tant du côté de la maîtrise de la langue / des langues et des disciplines que de celui du goût et de la sécurité des apprentissages ? En quoi permet-elle de conforter l'ensemble des compétences en langue ? Comment, enfin, une telle méthode pourraitelle bénéficier à davantage de classes, de quelle manière, et à quel coût?

Gauthier Couffin s'inscrit dans la continuité des propos de Pierre Escudé et examine dans un premier temps les enjeux d'un traitement des langues dans le continuum des langues romanes. Il analyse comment le travail sur l'intercompréhension peut permettre une meilleure prise en compte des biographies langagières des élèves et aussi en quoi cela constitue un pas vers le développement d'une éducation plurilingue

9 L'article de Jordi Cicres, Maria-Dolors de Ribot et Sívia Llach, se focalise sur la didactique intégrée des langues en Catalogne (Espagne). Le curriculum de l'Educación Primaria de Cataluña comprend l'étude de trois langues (le catalan, le castillan et une langue étrangère, majoritairement l'anglais) ainsi qu'une discipline appelée 
"Structures linguistiques communes " consacrée à l'étude des aspects linguistiques communs aux différentes langues. Les auteurs proposent une série d'activités pensées pour travailler en interrelations les aspects grammaticaux de ces trois langues.

10 Luci Nussbaum se propose d'illustrer la manière dont les ressources plurielles interviennent dans les interactions en classe, en même temps qu'elles constituent des outils indispensables dans le processus d'apprentissage. Son analyse s'appuie sur l'étude de trois cas de pratiques plurilingues enregistrées à l'école primaire en Catalogne (Espagne). Puis, elle s'interroge sur les orientations qui pourraient inaugurer une nouvelle étape de la didactique des langues.

\section{Partie 2 - Formation des enseignants au plurilinguisme}

11 Francis Troyan décrit le développement de la conscience langagière chez une enseignante américaine d'espagnol qui intègre la théorie du genre dans son enseignement de la langue à des élèves de $9 / 10$ ans (4th grade). Partant du constat que la conscience langagière est un élément essentiel de l'éducation plurilingue, l'auteur examine l'influence de la théorie du genre (éclairée par la linguistique systémiquefonctionnelle) sur les pratiques de l'enseignante. Il en tire des conclusions pour former des enseignants capables de développer l'identité plurilingue des élèves.

12 La recherche de Montserrat Fons et Juli Palou expose un processus de formation où les enseignants participants travaillent dans des environnements multiculturels et multilingues. Ce processus est basé sur la réflexivité dialogique, créant des espaces discursifs générés par des histoires de vie linguistique, des interviews, des groupes de discussion et des auto-confrontations. Les résultats de cette recherche montrent que les représentations émergent lorsque l'on met en place des processus d'interprétation dans l'action et dans le discours sur l'action.

13 L'article d'Annemarie Dinvaut s'intéresse aux écueils sur lesquels butent les apprenants lorsqu'il s'agit de rencontrer et comprendre l'autre et ses cultures. Quels dispositifs de formation concevoir pour favoriser des démarches d'appropriation et de réflexion chez les apprenants en langues-cultures? A. M. Dinvault présente un dispositif pluriel mis en place auprès d'étudiants en master FLE, qui privilégie le recours aux stratégies métalinguistiques et métacognitives ainsi qu'aux compétences plurilingues et fait une place à la dimension sensorielle de l'apprentissage des langues.

\section{Partie 3 - Contributions complémentaires}

Nous avons souhaité clore ce numéro par deux articles qui, s'ils sont essentiellement descriptifs, ont pour intérêt de nous renseigner sur des environnements nationaux/ régionaux particuliers et d'apporter un éclairage sur les situations alsacienne et slovaque en matière d'éducation aux langues. Leurs auteurs, impliqués dans l'enseignement des langues à des titres divers, nous invitent à partager leur compréhension de dispositifs (institutionnels, pédagogiques) existants, contribuant ainsi à ouvrir encore le champ des perspectives.

15 À partir de l'enseignement bilingue existant en France (modèles, enseignants, publications en didactique), Anémone Geiger-Jaillet et Gérard Schlemminger font un bref 
historique de l'enseignement et la formation bilingues du second degré en Alsace. Ensuite, les deux auteurs présentent le nouveau dispositif de formation continue "Enseigner sa discipline en allemand", ses contenus et les choix faits en Alsace en 2013. L'objectif de ce diplôme est de permettre d'alimenter le vivier d'enseignants formés pour assumer cette double mission : enseigner une discipline dans une langue autre que le français dans les classes ou sections bilingues et les sections européennes ou internationales.

À partir d'un bref aperçu historique, Anna Butašová fait un exposé général de la situation de l'enseignement des langues en Slovaquie et présente le caractère particulier du plurilinguisme slovaque issu de la grande diversité linguistique et culturelle du pays. Si l'auteure regrette que la problématique du plurilinguisme se voit souvent réduite à l'offre des langues étrangères enseignées, elle loue et décrit le projet ambitieux de formation des maîtres slovaques, mené en six langues et visant à préparer les enseignants à réfléchir sur les enjeux de développement de la compétence plurilingue des élèves.

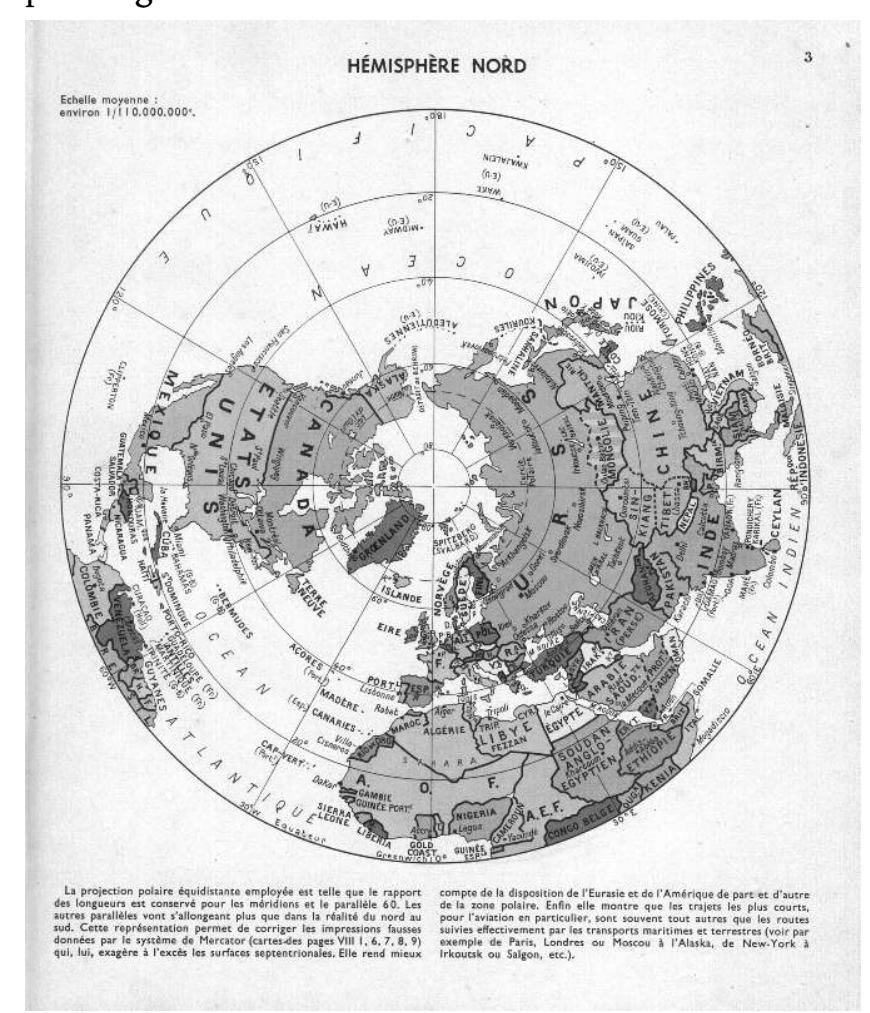

Fallex, M. ; Gilbert, A. (1953). Nouvel Atlas classique. Paris : Delagrave, p. 3. (Cedrhe)

\section{AUTEURS}

\section{PATRICIA MONJO}

Formatrice, Faculté d'Education - Université Montpellier 2 Dipralang, EA739 / Lirdef, EA 3749 


\section{RITA PEIX}

Formatrice, Faculté d'Education - Université Montpellier 2 Dipralang, EA739 / Lirdef, EA 3749 